\title{
Search for pair- and single-production of vector-like quarks in final states with at least one $Z$ boson decaying into a pair of electrons or muons in $p p$ collision data collected with the ATLAS detector at
} $\sqrt{s}=13 \mathrm{TeV}$

\section{Elena Freundlich*t, On behalf of the ATLAS Collaboration \\ Technische Universität Dortmund \\ E-mail: elena.freundlich@cern.ch}

\begin{abstract}
A search for pair- and single-production of vector-like quarks $T$ and $B$ with a leptonically decaying $Z$ boson is presented. The data were collected in $p p$ collisions at $\sqrt{s}=13 \mathrm{TeV}$ with the ATLAS detector at the LHC, corresponding to an integrated luminosity of $36.1 \mathrm{fb}^{-1}$. Final states are required to contain a $Z$ boson reconstructed from a same-flavor pair of leptons with opposite electric charge and a third generation quark. The analysis targets both pair- and single-production of vector-like quarks and no excess above the Standard Model expectation is found. Lower mass limits on vector-like $T$ and $B$ quarks at 95\% confidence level are derived and limits on the coupling to Standard Model quarks are set for single-production.
\end{abstract}

Sixth Annual Conference on Large Hadron Collider Physics (LHCP 2018)

4-9 June 2018

Bologna, Italy

\footnotetext{
* Speaker.

${ }^{\dagger}$ This work is supported by the Konrad-Adenauer-Stiftung.
} 


\section{Introduction}

When searching for physics beyond the Standard Model (SM), Composite Higgs [1, 2] and Little Higgs [3,4] models play an important role. These models predict vector-like quarks, whose right- and left-handed parts have the same transformation properties with respect to the weak isospin group SU(2). This analysis [5] at the ATLAS experiment [6] at the LHC focuses on the search for pair- and single-production of vector-like $T$ and $B$ quarks with charges $q_{T}=\frac{2}{3} e$ and $q_{B}=-\frac{1}{3} e$. For the vector-like quark decays the assumption is made that only decays to a $W, Z$ or $H$ boson and a third generation quark are possible. The final state is required to contain a $Z$ boson reconstructed from a lepton pair $\ell^{+} \ell^{-}(\ell=e, \mu)$. Figure 1 shows Feynman diagrams for both processes under study, one mediated by the weak and one by the strong force.
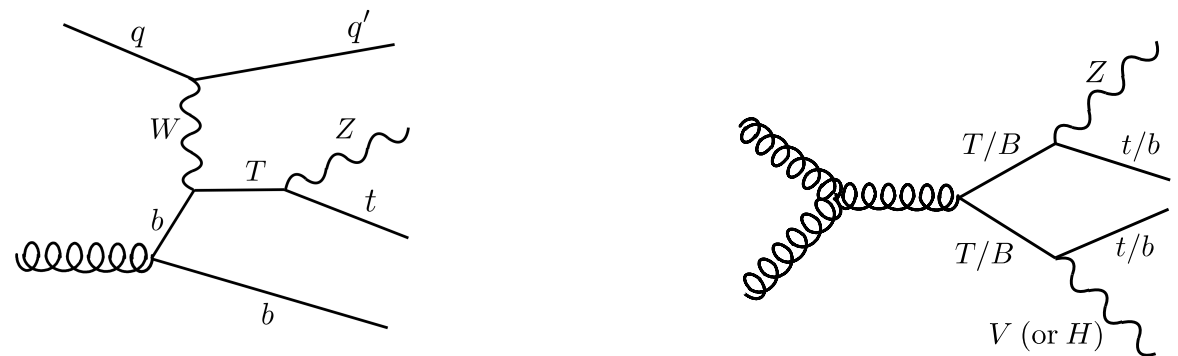

Figure 1: Feynman diagrams for single-production (left) and pair-production (right) of vector-like quarks with at least one $Z$ boson in the final state.

\section{Analysis strategy}

The final states presented in Figure 1 contain different multiplicities of leptons, large- $R$ jets with radius $R=1.0, b$-tagged small- $R$ jets with $R=0.4$ and high- $p_{\mathrm{T}}$ objects alongside other discriminating features.
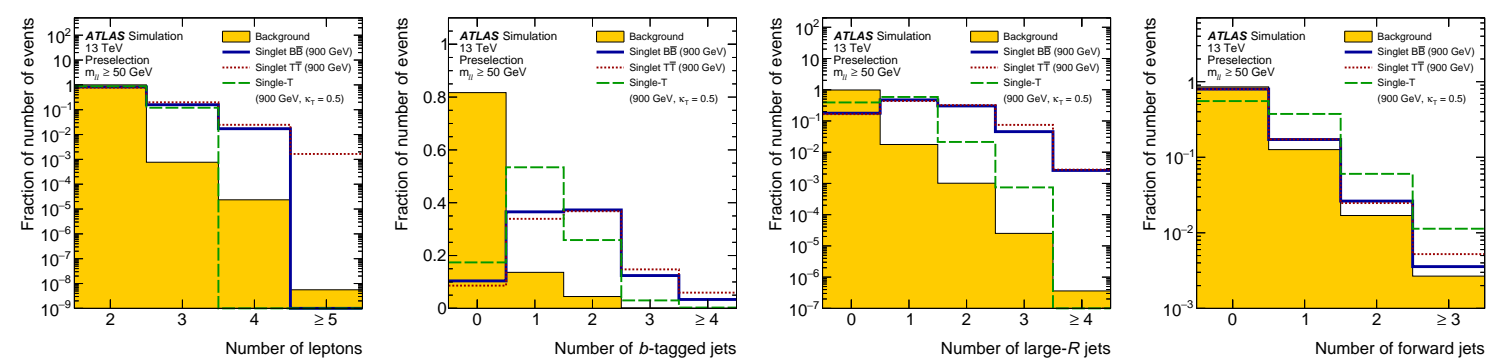

Figure 2: Normalized distributions of the lepton multiplicity, the number of $b$-tagged jets, the large- $R$ jet multiplicity and the forward jet multiplicity (from left to right) [5].

Figure 2 shows the distributions used for splitting the analysis into three orthogonal channels for pair-production, while there are two different channels for single-production. Channels with two leptons have a higher signal efficiency, while the background rejection is better for channels with at least three leptons. A requirement of at least one $b$-tagged small- $R$ jet rejects a lot of background and can be extended to at least two for channels with a large number of events. Furthermore, the number of large- $R$ jets discriminates well between signal and background and is used in the pair-production (PP) dilepton $(2 \ell)$ channels. For the single-production channels, the number 
of forward jets is required to be at least one, removing a substantial amount of background. To summarize, five different channels are defined: These are PP $2 \ell$ with $\leq 1$ large- $R$ jet or $\geq 2$ large- $R$ jets, $\mathrm{PP} \geq 3 \ell$, and for single-production (SP) $2 \ell$ and $\geq 3 \ell$. The main backgrounds are $Z+$ jets (PP $2 \ell 0-1 \mathrm{~J}$, PP $2 \ell 2 \mathrm{~J}, \mathrm{SP} 2 \ell$ ), $t \bar{t}$ (PP $2 \ell 0-1 \mathrm{~J}, \mathrm{PP} 2 \ell 2 \mathrm{~J}), t \bar{t}+X$ (PP $2 \ell 2 \mathrm{~J}, \mathrm{PP} \geq 3 \ell, \mathrm{SP} 2 \ell, \mathrm{SP} \geq 3 \ell$ ) and diboson events ( $\mathrm{PP} \geq 3 \ell$, SP $2 \ell, \mathrm{SP} \geq 3 \ell$ ).

Five channels are optimized individually and a final discriminant is chosen. Either the reconstructed vector-like quark mass $m_{Z b}$ or $m_{Z t}$ or a scalar sum of object momenta is chosen $\left(H_{\mathrm{T}}\right.$ for jets and $S_{\mathrm{T}}$ for leptons+jets). In Figure 3 and Figure 4 the final discriminants for each channel are illustrated. Data and Monte Carlo expectation agree well within the uncertainties.

Each discriminant is used in a statistical analysis in order to test for a discovery or set limits on the vector-like quark mass or the production cross-section times branching ratio $\sigma \times B R$. For the statistical analysis a binned profile likelihood fit is used based on the $C L_{S}$ method.
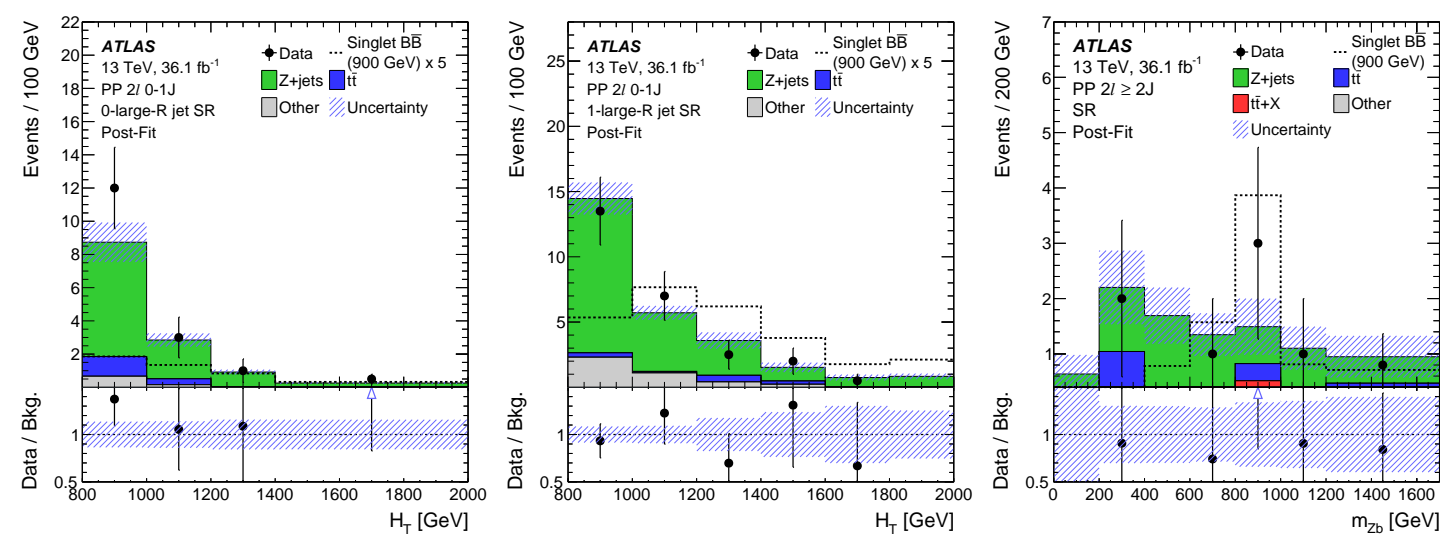

Figure 3: Final discriminants for the PP $2 \ell$ channels showing $H_{T}$ (left and center) and $m_{Z b}$ (right) [5].
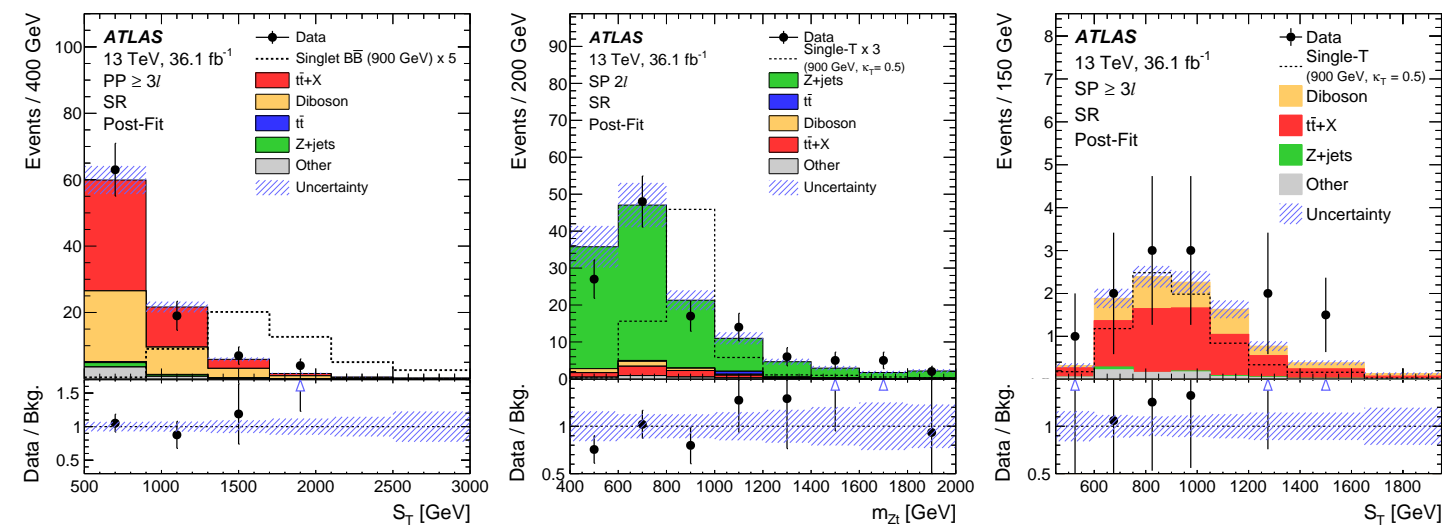

Figure 4: Final discriminants for the $\mathrm{PP} \geq 3 \ell$ channel (left) and for the single-production dilepton (center) and trilepton (right) channels [5].

\section{Results}

After performing the profile likelihood fit with all systematic uncertainties taken as nuisance parameters, no significant excess above the SM expectation is observed. Therefore, 95\% confi- 
dence level $(C L)$ limits are derived on $\sigma \times B R$ and the couplings to the SM quarks. In Table 5 the limits for PP are listed for the doublet models (with $B R(T / B \rightarrow t / b)=50 \%$ ).

Instead of focusing on a specific signal model the whole branching ratio plane can be scanned under the assumption that all $B R$ s add up to one. Figure 6 shows this scan and clearly illustrates the largest sensitivity in the $Z$ corner.

\begin{tabular}{c|c|c|c|c}
\hline Model & $\begin{array}{c}2 \ell 0-1 \mathrm{~J} \\
\text { exp. }\end{array}$ & $\begin{array}{c}2 \ell \geq 2 \mathrm{~J} \\
\text { exp. }\end{array}$ & $\begin{array}{c}\geq 3 \ell \\
\text { exp. }\end{array}$ & $\begin{array}{c}\text { Combination } \\
\text { obs. (exp.) }\end{array}$ \\
\hline$T \bar{T}$ doublet & $820 \mathrm{GeV}$ & $1100 \mathrm{GeV}$ & $1150 \mathrm{GeV}$ & $1210(1210) \mathrm{GeV}$ \\
\hline$B \bar{B}$ doublet & $1000 \mathrm{GeV}$ & $1070 \mathrm{GeV}$ & $880 \mathrm{GeV}$ & $1140(1120) \mathrm{GeV}$ \\
\hline
\end{tabular}

Figure 5: Expected exclusion limits on the vector-like quark $T$ or $B$ masses for each individual channel. Expected and observed limits are shown for the combination [5].
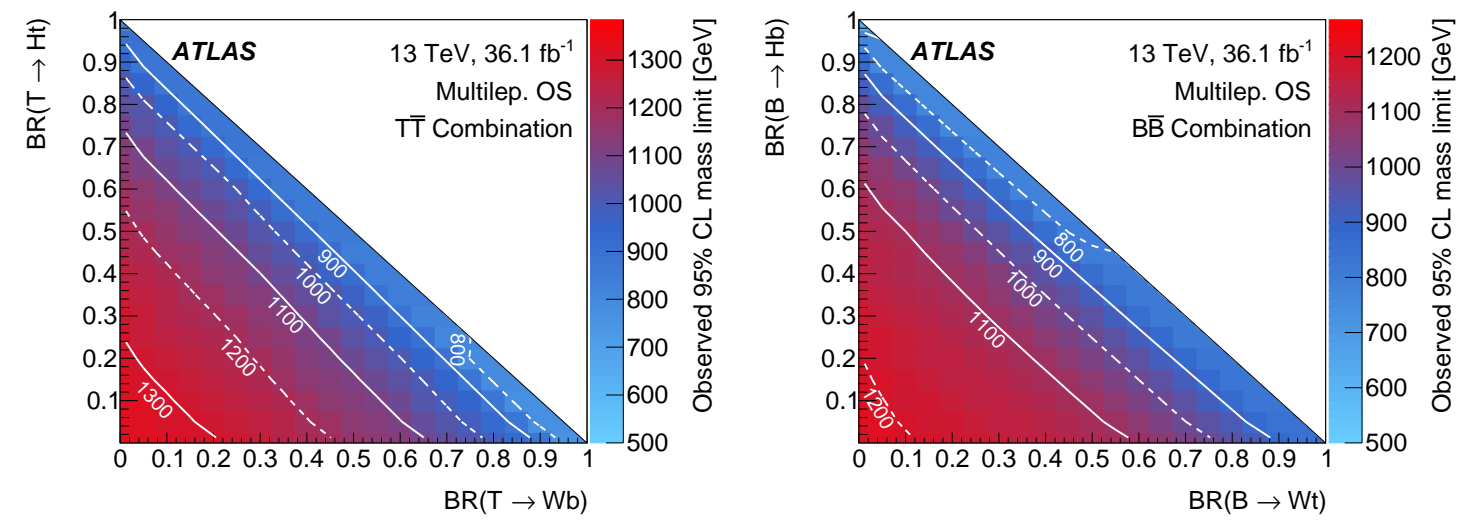

Figure 6: Two-dimensional limits in the branching ratio plane for vector-like $T$ (left) and $B$ (right) showing the PP combination limits [5].
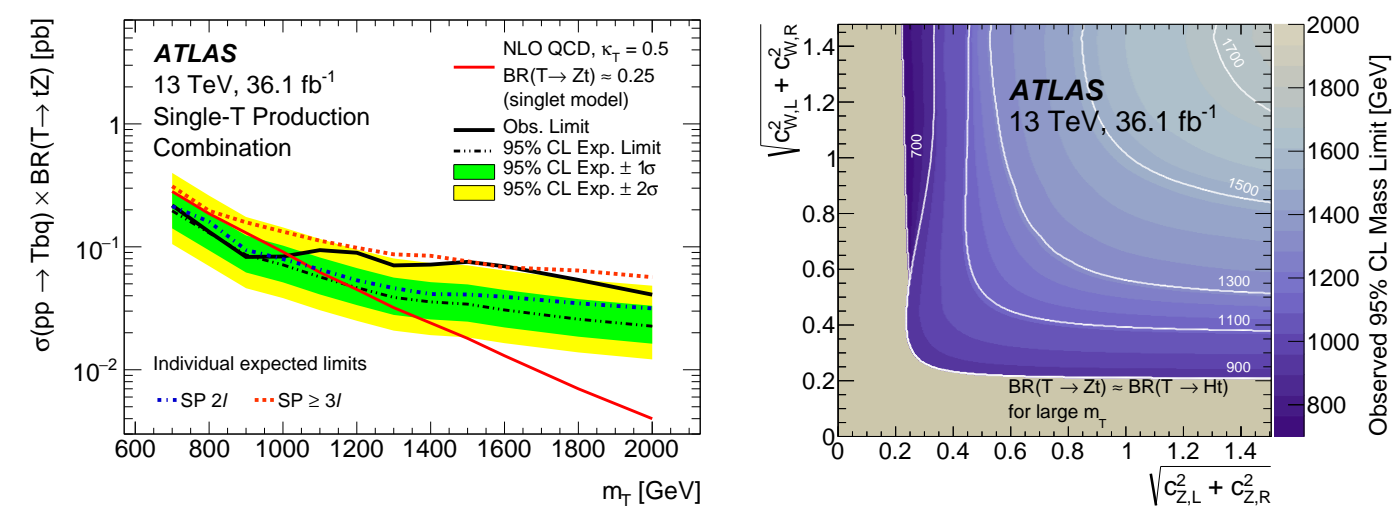

Figure 7: Expected and observed 95\% CL limits on $\sigma \times B R$ depending on the vector-like $T$ mass $m_{T}$ for the individual SP channels and their combination for a coupling of $\kappa_{\mathrm{T}}=0.5$ [7] (left). Observed lower limits on $m_{T}$ for SP combination as a function of the coupling $c_{W}$ and $c_{Z}$ (in the high- $m_{T}$ limit) [5] (right).

For single-production 95\% CL limits are set on $\sigma \times B R$ with a nominal coupling $\kappa_{\mathrm{T}}=0.5$ introduced in the model in Ref. [7]. In Figure 7 the limits are shown for both SP channels and 
their combination. The value of $\kappa_{\mathrm{T}}=0.5$ corresponds to $c_{W}=\sqrt{c_{L, W}^{2}+c_{R, W}^{2}}=0.45$, a coupling in another model from Ref. [8]. The coupling $c_{W}$ can then be reinterpreted in terms of a mixing angle $\left|\sin \left(\Theta_{L}\right)\right|$ [9] between $T$ and the SM top quark. In Figure 8 (left) the exclusion of the $c_{W}$ parameter space depending on the vector-like $T$ mass $m_{T}$ is shown for the singlet model. All values above the observed limit are excluded. For the mixing angle interpretation in Figure 8 (right) mixing angles within the contour are excluded. Furthermore, more generalized limits are set in couplingmass space by assuming $B R(T \rightarrow Z t) \approx B R(T \rightarrow H t)$ in the high-mass limit $m_{T}$. Subsequently, $T$ masses as a function of the coupling to $W, Z$ and $H$ can be excluded. The corresponding exclusion limit plot can be seen in Figure 7 (right).
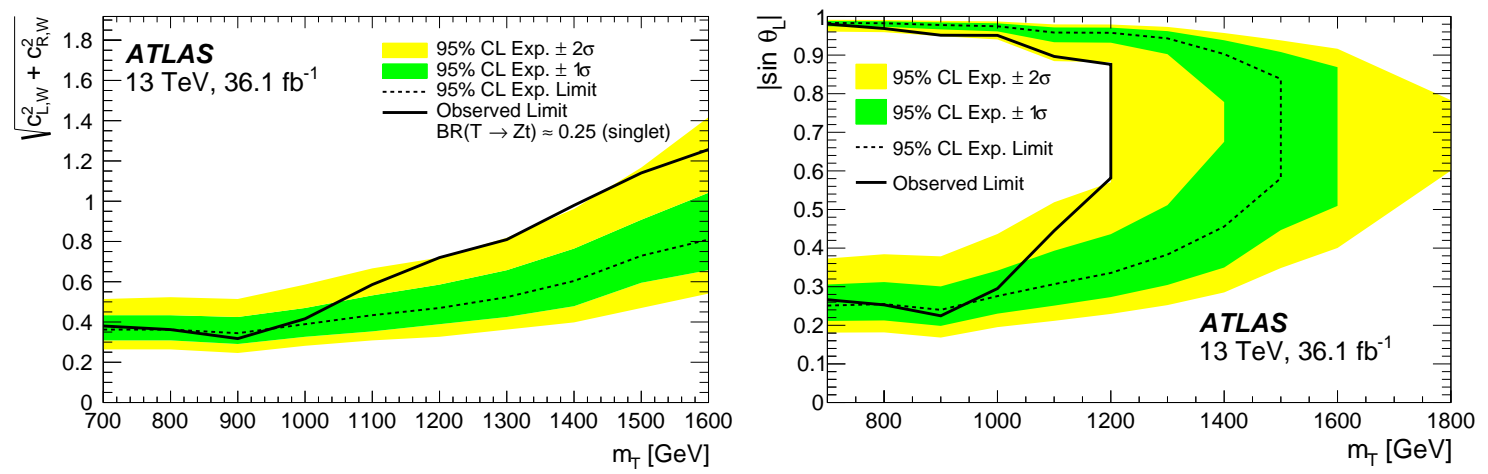

Figure 8: Expected and observed 95\% CL limits for the SP combination on the coupling $c_{W}$ of $T$ to SM particles in the singlet model (left) and on the mixing angle $\left|\sin \left(\Theta_{L}\right)\right|[5]$.

\section{Conclusion}

A search for vector-like $T$ (in pair- and single-production) and $B$ quarks (in pair-production) was presented with data collected at the ATLAS experiment at the LHC. No excess above the SM expectation was found and limits on $\sigma \times B R$ as well as different couplings and mixing angles could be set. The large number of high- $p_{\mathrm{T}}$ objects motivate the use of boosted techniques like large- $R$ jets. Single and pair-production channels were combined, increasing the overall sensitivity.

\section{References}

[1] D. B. Kaplan, H. Georgi, and S. Dimopoulos, Composite Higgs scalars, Phys. Lett. B 136 (1984) 187.

[2] K. Agashe, R. Contino, and A. Pomarol, The minimal composite Higgs model, Nucl. Phys. B 719 (2005) 165, arXiv: hep-ph/0412089.

[3] N. Arkani-Hamed, A. Cohen, E. Katz, and A. Nelson, The Littlest Higgs, JHEP 07 (2002) 34, arXiv: hep-ph/0206021.

[4] M. Schmaltz and D. Tucker-Smith, LITTLE HIGGS THEORIES, Ann. Rev. Nucl. Part. Sci. 55 (2005) 229, arXiv: hep-ph/0502182.

[5] ATLAS Collaboration,

Search for pair-and single-production of vector-like quarks in final states with at least one $Z$ boson decaying into a pair of electrons or muons in pp collision data collected with the ATLAS detector at $\sqrt{s}=13 \mathrm{TeV},(2018)$, arXiv: 1806.10555.

[6] ATLAS Collaboration, The ATLAS Experiment at the CERN Large Hadron Collider, JINST 3 (2008) S08003.

[7] M. Buchkremer, G. Cacciapaglia, A. Deandrea, and L. Panizzi,

Model-independent framework for searches of top partners, Nucl. Phys. B 876 (2013) 376, arXiv: 1305.4172.

[8] O. Matsedonskyi, G. Panico, and A. Wulzer, On the interpretation of Top Partners searches, JHEP 12 (2014) 097, arXiv: 1409.0100.

[9] J. A. Aguilar-Saavedra, R. Benbrik, S. Heinemeyer, and M. Pérez-Victoria, Handbook of vectorlike quarks: Mixing and single production, Phys. Rev. D 88 (2013) 094010, arXiv: 1306.0572. 